\title{
Energy Scavenging for Monitoring of Overhead Power Line Networks
}

\author{
Thomas Keutel, Xinming Zhao, Olfa Kanoun \\ Chair for Measurement and Sensor Technology \\ Chemnitz University of Technology, Germany
}

*Thomas Keutel, +49-371/531-36523, thomas.keutel@etit.tu-chemnitz.de

\begin{abstract}
:
The monitoring of overhead power line networks is becoming increasingly more challenging because of the power distribution of renewable energy resources. The use of ambient energy is important for a maintenance free supply of a wireless monitoring network. Generally, the electromagnetic field resulting from the $A C$ current and the electrostatic field resulting from the AC voltage can be used. Nevertheless, the energy scavenging from electrostatic field shows decisive advantages because of availability, stability during operation and reliability.

In this contribution we show the feasibility for the realisation of an energy scavenging unit. Theoretical investigations and simulations show that the distribution of the electric field in the immediate neighbourhood of the conductor shows a high gradient to the environment which can be used. The evaluation under realistic conditions in a high voltage laboratory show that through optimisation of the scavenging element an improvement of the amount of energy can be realised.
\end{abstract}

Keywords: Energy Scavenging, Overhead Power Line, Electrostatic Energy Conversion, Wireless Sensor Network

\section{INTRODUCTION}

Overhead power lines play an important role for a long distant electric power transmission because of the low cost for the transmission compared with buried cables also in the future. Their monitoring is becoming increasingly more challenging because of the effective charging of the grid [1-6]. Furthermore the distribution of new developed renewable energy resources, for example offshore wind farms, takes the existing transmission grid to capacity limits [7]. The amount of transported energy is strongly dependent on environmental conditions like temperature or snow [3]. In order to maintain low installation and maintenance costs, a completely wireless sensor network is aspired within the BMBF-project ASTROSE. For the power supply of the wireless nodes a new approach for energy scavenging was developed to supply a device from the direct boundary field around the conductor.

\section{SENSOR NETWORK}

Theoretical investigations of the electrical and mechanical behaviour and also the charge of power lines show that the temperature of the conductor is one of the most important limiting parameter during operation of a power line network [3,8]. For example, is there any damage or any deterioration of the conductor, the resistance $\left(R_{\text {cond }}\right)$ increases together with the temperature because of power losses $\left(P_{l}\right)$ :

$$
P_{I}=R_{\text {cond }} \cdot l_{\text {trans }}^{2}
$$

Also in cases of critical high charge $\left(I_{\text {trans }}\right)$ the temperature increases (s. equation (1)). Considering these facts, leads to the necessity of a monitoring system for measuring the local temperature of the conductor surface. 
Within the BMBF-project ASTROSE the development of a completely wireless and autonomous sensor network is aspired [5]. The system consists of widely distributed self configurable sensor nodes which measure the temperature of the conductor surface and the bending of the wires. Significant data are transmitted from the sensor nodes to a central gateway located near the transformer station (s. figure 1.) For supplying the distributed sensor nodes conventional strategies failed because of the demand of a long time reliability and maintenance-free operation.

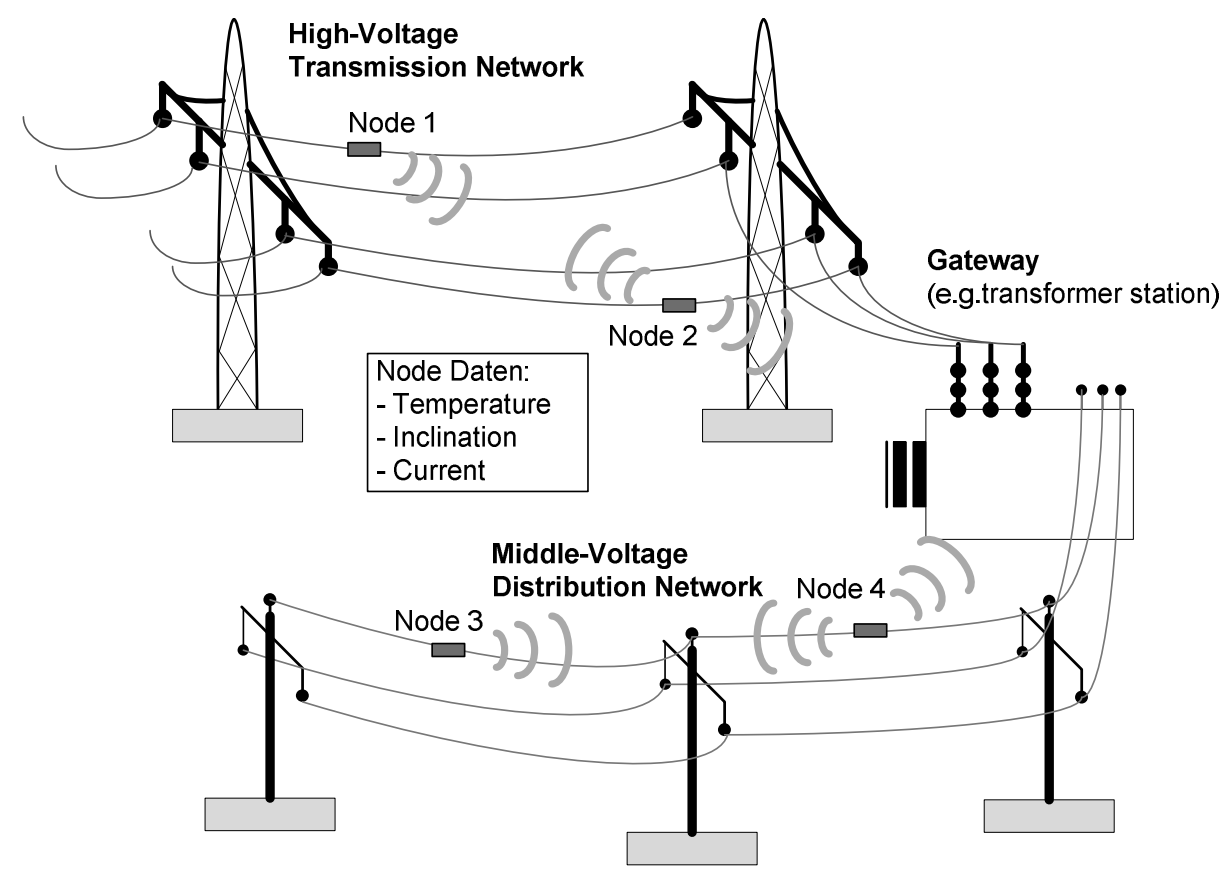

Figure 1. Principe of a wireless monitoring system

Several possibilities to supply the sensor nodes by ambient energy sources were investigated. Generally, there are two main possibilities for the conversion of electrical energy which can be considered at an overhead power line [1]. The most important one are the electromagnetic field resulting from the AC current and the electrostatic field resulting from the AC voltage. The availability, the stability and the reliability of the $A C$ voltage in relation to the $A C$ current during operation or failures of the transmission network, lead to the result, that the voltage, the electrical field, is the favourite energy source for supplying an electrical system directly mounted at the conductor of a overheat power line.

In the next sections, we will explain the theoretical background and describe some solutions allowing scavenging energy from the electrostatic field of the power line. Different possibilities will be compared and experimentally evaluated.

\section{THEORETIC CONSIDERATIONS AND SIMULATIONS}

The fundamental of the theoretical investigation [9] of the energy conversion using the electrostatic field represents the following equation in differential:

$$
\operatorname{rot} \vec{E}=\nabla \times \vec{E}=-\frac{\partial \vec{B}}{\partial t}
$$

or in integral form:

$$
\oint_{C} \vec{E} \vec{d} s=\iint_{A} \frac{\partial \vec{B}}{\partial t} d \vec{A}
$$

Thereby $\vec{E}$ describes the electrical field vector, $\vec{B}$ the electro-magnetic field vector and $s$ and $A$ space expressions. By the fact of the low grid frequency $\left(f_{g}=50 \mathrm{~Hz}\right)$; the following discussion is carried out without time-depending expressions $(d / d t=0)$.

Another important term is equation (4), described the relation between material and field conditions. 


$$
\vec{D}=\varepsilon_{0} \varepsilon_{r} \cdot \vec{E}
$$

Thereby $\vec{D}$ describes the electrical flux density and $\varepsilon_{0}, \varepsilon_{r}$ the electrical-field constant and the relative static permittivity. The field distribution is described by equation (5)

$$
\operatorname{div} \vec{D}=\nabla \cdot \vec{D}=\rho
$$

and describes the fact, that the electrical field line strayed from positive up to negative potential. Inserting equation (4) and equation (5) results, following several mathematical simplifications, an expression for the electrical field intensity:

$$
\vec{E}=-\operatorname{grad} \varphi
$$

Thereby $\varphi$ describes the electrical potential. Based on an ideal rotationally symmetric cylindrical electrode (fig. 2) results for the electrical field distribution the following expression using cylindrical coordinates:

$$
\frac{1}{r} \frac{\partial}{\partial r}\left(r \frac{\partial \varphi}{\partial r}\right)=0
$$

Concerning the conditions of figure 2 results a solution of equation (7):

$$
\varphi(r)=U-\frac{U}{\ln \frac{R_{\infty}}{R_{i}}} \ln \frac{r}{R_{i}}, \quad R_{i} \leq r \leq R_{\infty}
$$

Equation (8) shows that the electrical potential $\varphi$ depends simply on the voltage and on the radial distance $r$. Figure 3 shows the potential behaviour of a cylindrical electrode.

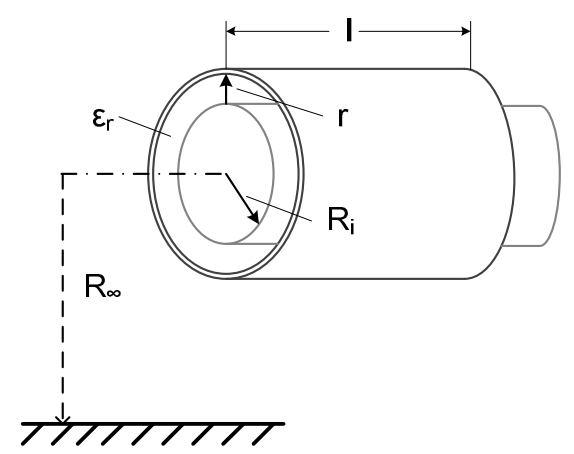

Figure 2. Structure of the cylinder electrode

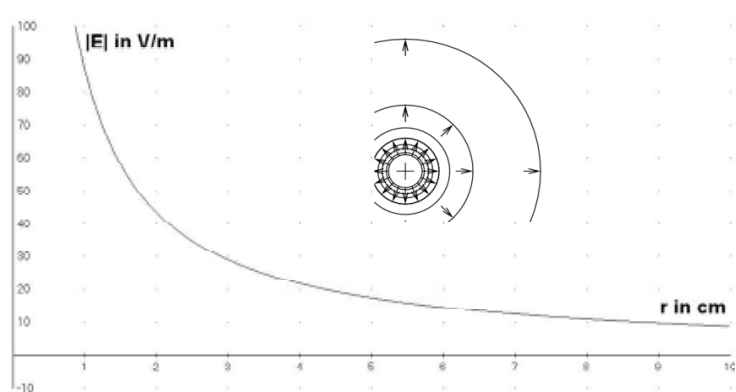

Figure 3. Gradient of the electrostatic field [5]

If there is a cylindrical electrode around the conductor at the distance $r$, a cylindrical capacitance is realised. This capacitance $\left(C_{c}\right)$ is characterised by the axial length $I$, the radial distances $R_{i}$ and $r$ and by the dielectric material $\varepsilon_{r}$ between the electrodes, shown in figure 2 . The capacitance is described by the equation:

$$
C_{c}=\frac{Q}{U_{C}}=\frac{\oint_{A} \vec{D} \cdot d \vec{A}}{\int \vec{E} \cdot d \vec{r}}=\frac{2 \pi \varepsilon_{r} \varepsilon_{0} \cdot l}{\ln \frac{\left(R_{i}+r\right)}{R_{i}}}
$$

The maximal storable energy $W$ of the capacitance is generally described by 


$$
W=\frac{1}{2} C \cdot U^{2}
$$

and for the cylindrical capacitance regarding equation (9):

and

$$
\begin{aligned}
& W_{c}=\frac{\pi l \varepsilon_{r} \varepsilon_{0} U^{2} \ln \frac{R_{i}+r}{R_{i}}}{\left(\ln \frac{R_{\infty}}{R_{i}}\right)^{2}} \\
& U_{c}=\frac{U}{\ln \frac{R_{\infty}}{R_{i}}} \cdot \ln \frac{R_{i}+r}{R_{i}}
\end{aligned}
$$

Thereby $U$ describes the voltage level of the grid and $U_{c}$ the resulting voltage of the coupling capacitance $C_{c}$. Equation (11) describes the electrical energy $W_{c}$, which can be stored, depends only on geometrical conditions of the capacitance $\left(R_{i}, r, I\right)$, the voltage level $(U)$ and of the dielectric material $\left(\varepsilon_{r}\right)$.

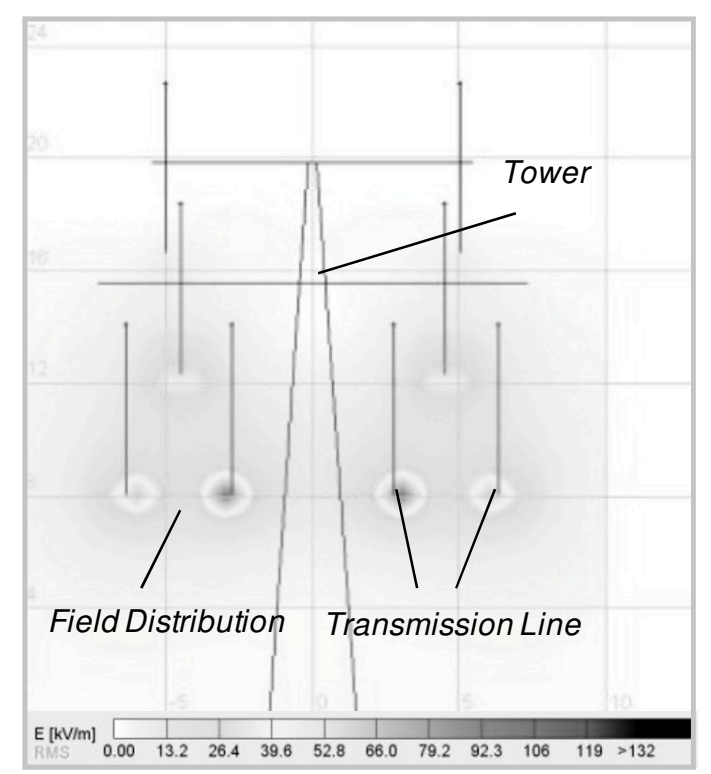

Figure 6. FEM simulation of electrostatic field around the power line in deployment [10]

Equations (11) and (12) describe a model for the behaviour of an energy converter using the electrostatic field, including all important parameters, such as $R_{\mathrm{i}}, r, I$ and $U$. This expression will serve as input for the construction of the sensor node, especially for the design of the couple capacitance of the converter.

For validating the theoretical results, FEM simulations were carried out to estimate the total energy availability [10]. The distribution of the electric field in the immediate neighbourhood of the conductor shows a high gradient to the environment of the line. This allows a direct energy scavenging (fig. 3 ). Figure 6 shows the field distribution and the field intensity concerning an exemplary tower of a overheat power line.

We expect that constructive aspects of the sensor node or of the coupling capacitance will influence the behaviour described above. Different constructive approaches will be compared therefore to ensure the optimal operation of the converter and with it the sensor node. 


\section{EXPERIMENTAL SETUP}

For the investigation of the energy availability from the electrostatic field two different test setups were realised as shown in figure 7.

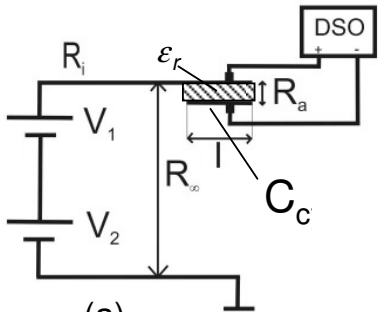

(a)

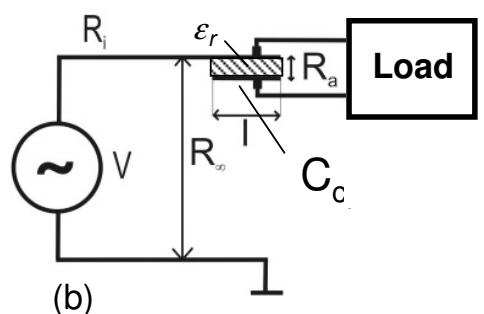

Figure 7. Test setup for (a) stationary case (b) realistic case [11]

For stationary investigations a test setup was realised by a serial connection of two voltage sources $\left(V_{1}\right.$, $V_{2}$ ) for a maximum voltage of $U_{D C}=400 \mathrm{~V}$ (fig.7a). For realistic measurements a test setup, shown in figure $7 \mathrm{~b}$, was realised for investigations in a high voltage laboratory at voltages up to $U_{A C, \max }=220 \mathrm{kV}$. As energy converter different cylindrical capacitors $\left(C_{c}\right)$ around an exemplary conductor of an $110 \mathrm{kV}$ overheat power line were realised.

Different scavenging setups were designed by using electrodes at different positions in the neighbourhood of the conductor (Figure 8). The simplest setup (setup 2) is using only one capacitance between the conductor and a surface electrode of the isolation (a thin solid dielectric material $\left(\varepsilon_{r}\right)$ ). In setup 1 an additional isolation was used in order to realise a second capacitance in series. In Setup 3 a parallel connection of setup 1 and setup 2 was carried out.

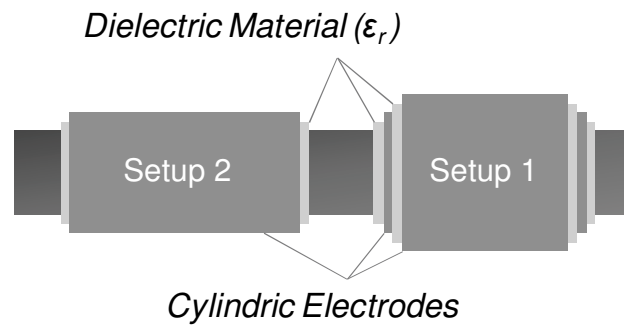

Figure 8. Considered cylindrical capacitances

\section{EXPERIMENTAL RESULTS}

For the test of the designed energy scavenger $C_{\mathrm{c}}$ an electric circuit was developed including a rectifier, a storage unit and a load consisting of a resistance and a diode. The diode illuminates by a certain voltage at the storage unit $C$. Measurements were carried out in a high voltage laboratory. The measured capacitances of the test setups 1,2 and 3 are in the range between 400 and $1200 \mathrm{nF}$, as can be seen in figure 10 [11].

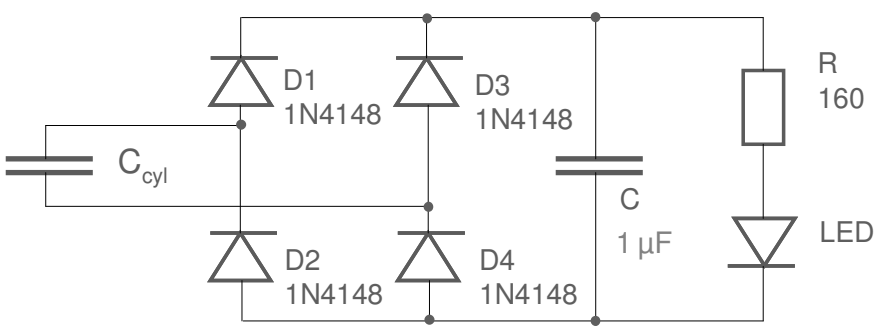

Figure 9. Test circuit for the energy scavenging unit [11] 
In figure 10 the minimal voltage at the conductor for diode illumination is shown for the different setups. Setup 3 is able to supply the test diode at even lower voltages and should be therefore preferred.

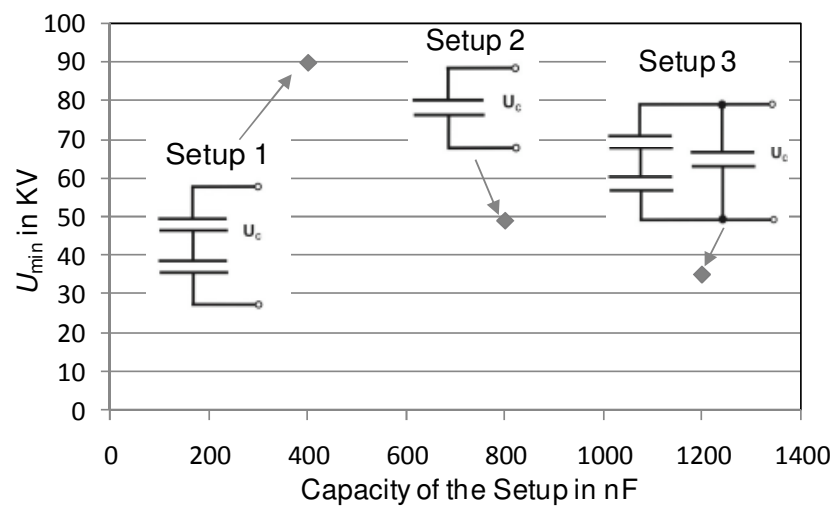

Figure 10. Minimal Voltage in dependence of the energy scavenging setup [11]

\section{CONCLUSION}

In this contribution fundamental investigations for the supply of wireless sensor nodes applied directly at the surface at an overhead power line. The investigations show that an electrostatic based energy scavenging should be preferred. Simulations and theoretical investigations have shown that the distribution of the electric field in the immediate neighbourhood of the conductor has a high gradient to the environment of the line and allows energy scavenging.

The feasibility of this kind of energy scavenging is demonstrated by an experimental setup. Different structures of energy scavenging elements were compared to each other. The setup 3 shows better properties.

The next steps will be the design of a power supply module able to supply a temperature sensor, an inclination sensor and a wireless communication stack. Some challenges will be the overvoltage protection of the module, the design of the scavenging element consideration system packaging and the harsh environment application.

\section{REFERENCES}

1. Zangl, Bretterklieber, Brasseur: „Energy Harvesting for Online Condition Monitoring of High Voltage Overhead Power Lines“, IEEE Instrumentation and Meassurement Techology Conference, 2008

2. Shoureshi, Lim, Sarusi: "Smart diagnostic system for high voltage electric transmission lines", American Society of Mechanical Engineers, Dynamic Systems and Control Division (Publication) DSC, 2006

3. Muhr, Pack, Jaufer, Haimbl, Messner: "Experiences with the Weather Parameter Method for the use in overhead line monitoring systems", Elektrotechnik und Informationstechnik 125 (12), pp. 444-447, 2008

4. Krco, Tsiatsis: "Mobile Network Supported Wireless Sensor Network Services", Mobile Adhoc and Sensor Systems, IEEE Internatonal Conference on, MASS 8.-11. Oct. 2007

5. Shangwei, Xiaobu: "Exploring hierarchy architecture for wireless sensor networks management", Wireless and Optical Communications Networks, IFIP International Conference on, 2006

6. Cheng, Niu, Li: "The reliability evaluation method of high voltage overhead transmission lines", Proceedings of 2008 International Conference on Condition Monitoring and Diagnosis, art. no. 4580351, pp. 566-569, CMD 2008

7. Jarass, Obermair: "Netzeinbindung von Windenergie: Erdkabel oder Freileitung?", Zeitschrift für Energiewirtschaft, vol. 6, pp. 398-403, 2005

8. Mühl: "Berechnung der Grenzen von Freileitungsparametern sowie Ableitung und Bewertung der Abhängigkeiten"; Student Research Project, Chemnitz University of Technology, 2007

9. Philippow: "Grundlagen der Elektrotechnik", ISBN: 978-3341012413, 2000

10. Peppelyashev: "Theoretical Investigations of Energy Scavenging for a Power Line Monitoring System", Student Research Project, Chemnitz University of Technology, 2007

11. Kurz: "Practical Investigation to Energy Scavenging from Electrostatic and Magnetic Field of a Power Line", Student Research Project, Chemnitz University of Technology, 2007 